\title{
Significance of cytodiagnosis in primary carcinoid tumor of the middle ear: A case report
}

\author{
KIMINOBU SATO ${ }^{1,2}$, RYOTA MIHASHI ${ }^{1}$, TAKASHI KURITA ${ }^{1}$, HIROHITO UMENO ${ }^{1}$, \\ TOMOKO YOSHIDA ${ }^{3}$, AKIHIKO KAWAHARA ${ }^{3}$, JUN AKIBA $^{3}$ and HIROHISA YANO ${ }^{2}$ \\ Departments of ${ }^{1}$ Otolaryngology-Head and Neck Surgery, ${ }^{2}$ Pathology and \\ ${ }^{3}$ Diagnostic Pathology, Kurume University School of Medicine, Kurume, Fukuoka 8300011, Japan
}

Received November 10, 2016; Accepted October 24, 2017

DOI: $10.3892 / \mathrm{ol} .2018 .8302$

\begin{abstract}
Primary carcinoid tumors of the middle ear are uncommon. Cytodiagnosis of the middle ear is not usually performed as a preoperative examination because of the anatomical structure of the middle ear. To the best of our knowledge, the present study reports for the first time a case of a carcinoid tumor of the middle ear, which was preoperatively diagnosed using cytodiagnosis, and also review current literature. A 22-year-old woman complained of left-sided otalgia. A subcutaneous tumor in the left middle ear was observed. Magnetic resonance imaging revealed that the tumor was primarily present in the tympanic cavity of the middle ear and extended to the mastoid antrum and mastoid cells of the middle ear and to the external acoustic meatus. Fine needle aspiration cy tology was performed from the tumor protruding into the external acoustic meatus. Cytologically, the tumor was composed of small uniform cells with small round nuclei and granular eosinophilic cytoplasm, arranged in a nested pattern. Tumor cells were immunocytochemically positive for synaptophysin and cytokeratin CAM5.2. These results indicated that this was a neuroendocrine tumor. No other tumor lesions were identified on computed tomography scans, and the tumor was considered to be the primary lesion. Tympanoplasty and mastidectomy were then performed and carcinoid tumor was diagnosed in the postoperative pathological diagnosis. To the best of our knowledge, the present study is the first to report on preoperative cytodiagnosis for carcinoid tumors of the middle ear.
\end{abstract}

Correspondence to: Dr Kiminobu Sato, Department of Otolaryngology-Head and Neck Surgery, Kurume University School of Medicine, 67 Asahi-machi, Kurume, Fukuoka 8300011, Japan E-mail: satou_kiminobu@med.kurume-u.ac.jp

Key words: carcinoid tumor, middle ear, cytodiagnosis, fine needle aspiration cytology

\section{Introduction}

Carcinoid tumors occur in various types of organs, the most frequent of which are identified in the digestive system (64.2\%). Primary carcinoid tumor of the middle ear is an extremely rare form of carcinoid tumor $(<0.7 \%)(1,2)$. Almost all cases of the disease are diagnosed via biopsy analysis, postoperative pathological examination, or transmission electron microscopy in atypical histopathological cases $(3,4)$. In carcinoid tumors of other organs, such as those of the digestive system and lungs, fine needle aspiration cytology (FNAC) is a routine preoperative examination. However, in carcinoid tumors of the middle ear, FNAC is not usually performed as a preoperative examination as there is a risk of complications due to the complex anatomical structure of the middle ear. To the best of our knowledge, there have been no previous reports on preoperative cytodiagnosis for carcinoid tumors of the middle ear.

The primary course of treatment is surgery in the majority of localized cases $(2,5)$; however, treatment for patients with unresectable metastatic disease may involve a combination of surgical resection and systemic therapy (e.g., a course of chemotherapy) (5). For patients with functional tumors, somatostatin analogues are the primary treatment regime, which aims to control symptoms caused by an excess of hormones including insulin gastrin, glucagon, vasoactive intestinal peptide and somatostatin (5). A recent study provided evidence that the choice of treatment options for patients with carcinoid tumors should increased and be based upon the extent, tumor proliferation rate, symptoms, histological grade and primary tumor site (5). Therefore, accurate clinical and/or histological diagnosis is required for patients with carcinoid tumor.

The present study discusses a primary middle ear tumor, which was preoperatively diagnosed as neuroendocrine tumor (NET) using cytology and was postoperatively diagnosed as a carcinoid tumor using histological examination.

\section{Case report}

A 22-year old Japanese woman with no previous history of disease complained of left-side otalgia and visited the outpatient clinic of Kurume University Hospital (Kurume, Japan). A physical examination revealed a subcutaneous tumor protruding into the left external acoustic meatus (the external 
auditory canal) (Fig. 1). An audiogram demonstrated conductive hearing loss. Gadolinium-enhanced T1-weighted magnetic resonance imaging (MRI) with low contrast enhancement (Fig. 2A) and diffusion weighted MRI with low intensity (Fig. 2B) revealed a tumor-like lesion occupied in the tympanic cavity of the middle ear, extending to the mastoid antrum and mastoid cells of the middle ear and to the external acoustic meatus. The lesion was considered to be a primary middle ear tumor, as it would never have extended to the mastoid antrum and mastoid cells if the tumor had originated from the external acoustic meatus. These imaging results were not able to lead to a definitive diagnosis, although they excluded the possibility of glomus tumor or cholesteatoma. Therefore, FNAC was performed.

The cytological findings revealed that the tumor was composed of cells, which exhibited small round nuclei with dispersed chromatin and granular eosinophlic cytoplasm, arranged in a nested pattern (Fig. 3A). These tumor cells were positive for synaptophysin (Fig. 3B) and cytokeratin CAM5.2 (Fig. 3C) and negative for tumor protein p63 and Wilms tumor 1 immunocytochemically; Ki-67 immunoreactivity was observed in $<1 \%$ of the tumor cells. These results indicated that the tumor may be NET, likely a carcinoid tumor. The smears were fixed in $95 \%$ ethanol at room temperature for one day and analyzed using Papanicolaou staining. Papanicolaou staining was performed as below.

Briefly, slides were washed with $70 \%$ ethanol for $3 \mathrm{~min}$ and then washed with tap water for 2 min. Slides were stained with Gill's hematoxylin (Muto Pure Chemicals Co., Ltd., Tokyo, Japan) at room temperature for $5 \mathrm{~min}$, rinsed with $1 \%$ hydrochloric acid alcohol for $7 \mathrm{sec}$, washed with tap water for $7 \mathrm{~min}$, and then twice with $95 \%$ ethanol for $1 \mathrm{~min}$ each. Slides were then stained using Orange G-6 (Ready to use dilution, Matsunami Glass Ind., Ltd. Osaka, Japan) solution (Matsunami Glass Ind., Ltd., Kishiwada, Japan) at room temperature for $3 \mathrm{~min}$, and washed twice with $95 \%$ ethanol for 1 min each. Slides were then stained using eosin A-50 solution (Matsunami Glass Ind., Ltd.) at room temperature for $4 \mathrm{~min}$, and washed twice with $95 \%$ ethanol for 1 min each. Slides were washed three times with $100 \%$ ethanol for 2 min each, immersed in xylene three times for 2 min each and mounted in Marinol mounting medium (Muto Pure Chemicals Co., Ltd.).

Laboratory examination revealed that serum 5-hydroxyindoleacetic acid (5-HIAA, normal range: 1.8-6.1 ng/ml) and vanillylmandelic acid (3.3-8.6 ng/ml), urine total catecholamine (normal range: $52.0-195.3 \mu \mathrm{g} /$ day) and 5-HIAA (normal range: $1.0-6.0 \mathrm{mg} / \mathrm{day}$ ) were within the normal reference ranges. Briefly, serum samples were dispensed, and deproteinised with $6 \%$ perchloric acid. Following centrifugation $\left(11,269 \times \mathrm{g}, 4^{\circ} \mathrm{C}, 17 \mathrm{~min}\right)$, the supernatant was extracted and used for analysis. Wakosil-II 5C18HG (cat. no. N 235-51001; Wako Pure Chemical Industries, Ltd., Osaka, Japan) and Wakosil-II 3C18HG (cat. no. N 238-50251, Wako Pure Chemical Industries, Ltd.) were used for 5-HIAA analysis. TSKgel ODS-80Ts (cat. no. N 0018151; Tosoh Bioscience, Tokyo, Japan) and Pegasil ODS (cat. no. N PG-ODS-1101-SPW; Senshu Scientific Co., Ltd, Tokyo, Japan) were used for vanillylmandelic acid. Serum 5-HIAA and vanillylmandelic acid samples were analyzed using a high liquid performance chromatography (HPLC) system (Shimadzu Corporation, Kyoto, Japan). Single voided urine samples were dispensed, and Wakosil-II 5C18HG (cat. no. N 232-51011; Wako Pure Chemical Industries, Ltd.) was used for urine 5-HIAA analysis, and Wakosil-II 5C18RS (cat. no. N 232-51371; Wako Pure Chemical Industries, Ltd.) and TSKgel CA-2 (cat. no. N 0018932; Tosoh Bioscience) were used for catecholamine analysis. Urine catecholamine and 5-HIAA samples were analyzed using an HPLC system, perfomed by Shimadzu Corporation.

There was no paraneoplasia, including diarrhea, suffusion or edema. No other tumor lesions in other organs were detected on computed tomography scans. Consequently, a diagnosis of primary carcinoid tumor of the middle ear was considered.

Since the tumor involved the mastoid anturm, mastoid cells and external auditory canal, two-staged tympanoplasty and mastiodectomy was performed. Since the boundary of the tumor in the tympanic cavity around the stapes was unclear, the incus was removed to allow for complete resection of the tumor. A second-stage operation was planned to prevent recurrence.

The tumor was histologically composed of small uniform cells with small round nuclei and granular eosinophilic cytoplasm, and was proliferating in trabecular and mosaic patterns (Fig. 4A and B). Immunoreactivity with synaptophysin was identified in tumor cells. On the other hand, immunoreactivity with chromogranin A and cluster of differentiation (CD)56 was not detected. Ki-67 index was identified in $<2 \%$ of the cells. The definitive diagnosis was carcinoid tumor.

Briefly, paraffin-embedded tissue samples were cut at $4 \mu \mathrm{m}$ thick, examined on a coated slide glass and labeled with the following antibodies: Mouse monoclonal anti-synaptophysin (cat. no. N 413831; ready to use dilution, Nichirei, Tokyo, Japan), mouse monoclonal anti-chromogranin A (cat. no. M0869; 1:400; Dako; Agilent Technologies, Inc., Santa Clara, CA, USA), mouse monoclonal anti-CD56 (cat. no. NCL-L-CD56-1B6; 1:200; Leica Microsystems, Ltd., Milton Keynes, UK) and mouse monoclonal anti-Ki67 (cat. no. M7240; 1:100; clone MIB1; Dako; Agilent Technologies, Inc.).

Two fully automated immunostainers, the BenchMark ULTRA system (Ventana Medical Systems, Inc., Tucson, AZ, USA) and the Bond-III system (Leica Microsystems, Ltd.), were used. The BenchMark ULTRA system was used for Ki-67 immunostaining. Briefly, each slide was heat-treated using the Ventana ULTRA cell conditioning 1 retrieval solution (EDTA; pH 8.5; Ventana Medical Systems, Inc. UAS) for $60 \mathrm{~min}$ at $95^{\circ} \mathrm{C}$, and then incubated with the $\mathrm{Ki}-67$ antibody (as aforementioned) for $30 \mathrm{~min}$ at $37^{\circ} \mathrm{C}$. The automated system used the Ventana UltraVIEW 3,3' diaminobenzidine (DAB) detection kit with a horseradish peroxidase (HRP) multimer as a secondary antibody, peroxidase inhibitor, DAB chromogen, $\mathrm{H}_{2} \mathrm{O}_{2}$, and copper (cat. no. 760-500; Ventana Medical Systems, Inc., USA). This detection kit is an indirect, biotin-free system for detecting mouse immunoglobulin (Ig)G, mouse IgM and rabbit primary antibodies. Each slide was incubated with the secondary antibodies for $30 \mathrm{~min}$ at $37^{\circ} \mathrm{C}$ and visualized with DAB and $0.04 \%$ hydrogen peroxide.

Immunostaining with synaptophysin, chromogranin A and CD56 were performed on the automated Bond-III system (Leica Microsystems, Ltd.) using onboard heat-induced antigen retrieval with epitope retrieval solution 2 (EDTA-based 


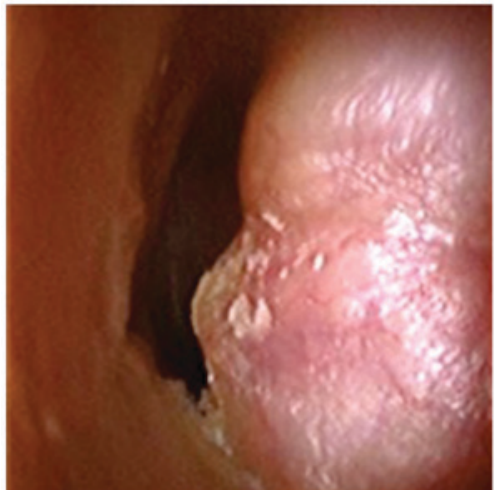

Figure 1. Endoscopic results of the left ear. Subcutaneous smooth tumor protruding into the external acoustic meatus without pulsating. The tympanic membrane was not identified.
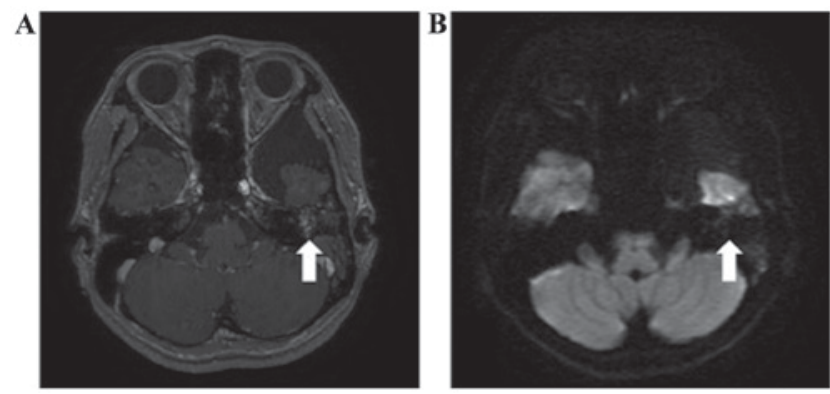

Figure 2. Axial magnetic resonance imaging of the temporal bone. The tumor lesion was primarily present in the tympanic cavity and extended to the mastoid antrum and mastoid cells. (A) Gadolinium enhanced T1-weighted imaging depicting tumor (arrow) with low contrast enhancement, excluding the possibility of vascular lesions, such as glomus tumors. (B) Diffusion-weighted imaging depicting low intensity tumor lesion (arrow), excluding the diagnosis of cholesteatoma.
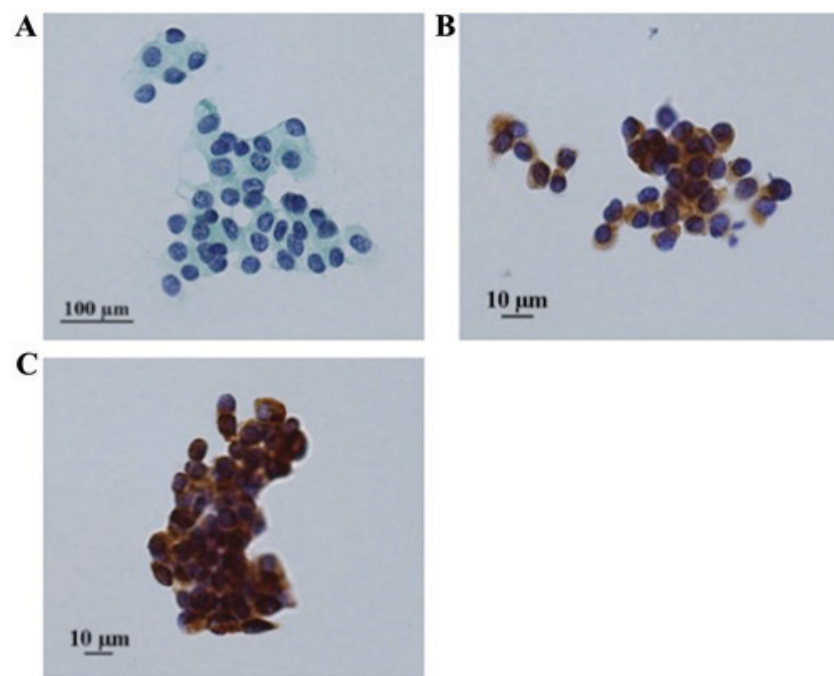

Figure 3. Cytological results. (A) Cytological results revealing that the tumor was composed of small uniform cells with small round nuclei and granular eosinophlic cytoplasm, arranged in a nested pattern. (B and C) Tumor cells were positive for (B) synaptophysin and (C) cytokeratin CAM5.2 immunocytochemically.

buffer; $\mathrm{pH}$ 9.0; Leica Microsystems, Ltd.) for $15 \min$ at $99^{\circ} \mathrm{C}$, and incubated with each primary antibody for $30 \mathrm{~min}$ at room
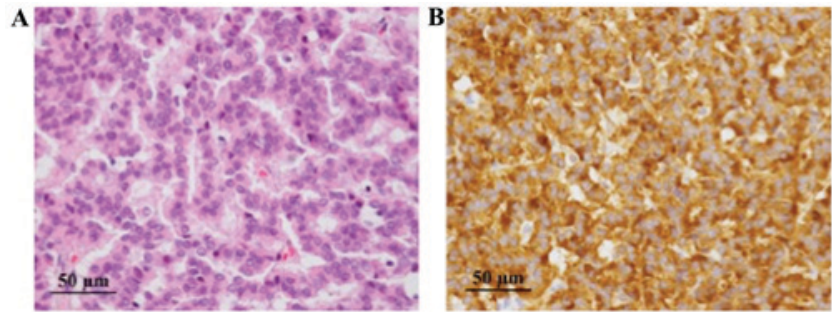

Figure 4. Pathological findings. (A) The tumor was histologically composed of small uniform cells with small round nuclei and granular eosinophilic cytoplasm, and was proliferating in trabecular and mosaic patterns (hematoxylin and eosin stain). (B) Immunohistochemical studies revealed positive for synaptophysin.

temperature. This automated system used a refine polymer detection kit (cat. no. TA9145; Leica Microsystems, Ltd.) with HRP-conjugated anti-mouse/rabbit IgG and goat polyclonal antibody as a secondary antibody, and was incubated for $30 \mathrm{~min}$ at room temperature. Slides were visualized using $\mathrm{DAB}$ and $0.1 \%$ hydrogen peroxide.

Prior to hematoxylin and eosin staining, slides were placed in xylene and hydrated by passing through alcohol $(70,80$, 90 and $95 \%$ alcohol for 2 min each). Slides were stained in hematoxylin (cat. no. 8650, Sakura Finetek Europe B.V., Flemingweg, The Netherlands) for 5 min, washed with tap water for $5 \mathrm{~min}$, and then stained with eosin (cat. no. 8659, Sakura Finetek Europe B.V.) for $10 \mathrm{~min}$ at room temperature.

At 8 months following the primary surgery, incus interposition ossiculoplasty was performed as the second-stage operation. The patient remained recurrence-free for 11 months during follow-up examinations. The patient provided written informed consent for inclusion in the present study.

\section{Discussion}

The middle ear is composed of the tympanic cavity, mastoid antrum and mastoid cells. The tympanic cavity is a tiny cavity containing auditory ossicles. FNAC of the middle ear is not a routine examination owing to the risk of complications, including ossicular disruption or bleeding. Krouse et al (6) reported that the tympanic membrane was retained but was reddened, thickened or bulging in almost all cases of carcinoid tumor of the middle ear. There are few reports of tumors of the middle ear that have invaded into the subcutaneous tissue of the external acoustic meatus $(3,7)$. Biopsies were attempted in these cases, although FNAC was not performed.

FNAC is a common examination for canal tumors of the external acoustic meatus (8). Mohan et al (9) reported a case of adenoid cystic carcinoma of the external acoustic meatus diagnosed by FNAC and discussed its utility. In subcutaneous tumor cases, an incisional biopsy is required to obtain adequate specimens; however, there is a risk of bleeding. As FNAC is relatively safe compared with biopsy, cytology can be a complementary or alternative examination for biopsy for tumors of the external acoustic meatus and the middle ear. In the present case, FNAC may be performed because the tumor protruded into the external auditory canal.

Carcinoid tumors of the middle ear were first described by Murphy et al (10) in 1980. Additional cases have been reported 
and their clinical and histopathological characteristics have also become clear. Carcinoid tumors of the middle ear exhibit the same histopathological and morphological characteristics as pulmonary and gastrointenstinal carcinoid tumors (11).

Morphological findings of carcinoid tumors are described in which the nuclei are round-to-oval with dispersed chromatin, and eosinophilic and granular cytoplasm (11). In Papanicolaou staining, aspirated specimens exhibit monotonous groups of well-preserved round cells or a mixture of round and spindle cells (12). Cytological features of NETs, including carcinoid tumor, often mimic small cell carcinoma and/or malignant lymphoma. It is important to distinguish carcinoid tumor from these neoplasms, as the clinical treatment is completely different. Immunohistochemical examination is useful for distinguishing these tumors.

Immunohistochemistry of neuroendocrine markers, including chromogranin A, synaptophysin and cluster of differentiation 56, is useful for making a diagnosis of NETs. There are several reports on the immunohistochemistry of carcinoid tumors (11-13). Immunocytochemistry was not commonly performed because the amount of cells is limited. However, with the advances in liquid based cytology (such as the BD Diagnostics SurePath test), performing immunocytochemistry has become easy. Indeed, immunocytochemistry was performed using liquid-based cytology material in the present case. Immunocytochemistry can be performed when carcinoids occur in unusual sites, including the middle ear or larynx $(4,14)$. As primary carcinoid of the middle ear is extremely rare, an entire body examination must be performed preoperatively to exclude the possibility of metastasis.

In the present case, stained with Papanicoloau stains, the nuclei of the tumor cells were round with dispersed chromatin and exhibited low-grade atypia, and the cytoplasm of the tumor cells were granular. On the basis of the morphological features, NETs, including a carcinoid tumor, were suspected, with immunocytochemical results supporting the cytological diagnosis.

In previous cases of carcinoid tumors of the middle ear, removal of the tumor by conventional tympanomastoidectomy improved the surgical procedure as tympanomastoidectomy enabled complete removal of the tumor within the middle ear $(6,15,16)$. It is not necessary to perform exploratory surgery in preoperatively diagnosed cases; therefore, it is important to achieve a diagnosis prior to surgery to perform radical surgery for the primary operation. Preoperative FNAC makes a substantial contribution to determining the operative method in cases of NETs, including carcinoid tumors of the middle ear.

In a previous cytological study, carcinoid and neuroendocrine cancer were diagnosed based on morphology and immunocytochemical analysis (17). In examinations that involve a comparison of the intraoperative cytologic diagnosis in small specimen with final histologic diagnosis of the resected specimen, the cytodiagnostic sensitivity and specificity are 86.7 and $98.7 \%$ respectively to diagnose pulmonary neuroendocrine tumor (18). As carcinoid tumors of the middle ear exhibit the same histopathological and morphological characteristics exhibited in pulmonary neuroendocrine tumors, FNAC with immunocytochemical analysis is a reliable examination for patients with carcinoid tumor of the middle ear.

To conclude, FNAC with immunocytochemistry is a reliable preoperative examination for NETs, including carcinoid tumors of the middle ear.

\section{Acknowledgements}

The authors would like to thank the members of SRL, Inc. (Tokyo, Japan) for providing information about the laboratory examination.

\section{Funding}

No funding was received.

\section{Availability of data and materials}

All data generated or analyzed during the present study, except those performed by an external organization, are included in this published article.

\section{Authors' contributions}

KS, RM, JA, HU and HY designed the study. KS wrote the initial draft of the manuscript. RM, TK, TY and AK contributed to analysis and interpretation of data. All authors revised the manuscript critically for important intellectual content and approved the final version of the manuscript.

\section{Ethics approval and consent to participate}

The patient provided written informed consent for inclusion in the present study.

\section{Consent for publication}

The patient provided written informed consent for publication of the present study.

\section{Competing interests}

The authors declare that they have no competing interests.

\section{References}

1. Soga J: Carcinoid and their variant endocrinomas. An analysis of 11842 reported cases. J Exp Clin Cancer Res 22: 517-530, 2003.

2. Godwin JD II: Carcinoid tumors. An analysis of 2,837 cases. Cancer 36: 560-569, 1975 .

3. Ramsey MJ, Nadol JB Jr, Pilch BZ and Mckenna MJ: Carcinoid tumor of the middle ear: Clinical features, recurrences, and metastases. Laryngoscope 115: 1660-1666, 2005.

4. Tamai S, Iri H, Maruyama T, Kasahara M, Akatsuka S, Sakurai S and Murakami Y: Laryngeal carcinoid tumor: Light and electron microscopic studies. Cancer 48: 2256-2259, 1981.

5. Kunz PL: Carcinoid and neuroendocrine tumors: Building on success. J Clin Oncol 33: 1855-1863, 2015.

6. Krouse JH, Nadol JB Jr and Goodman ML: Carcinoid tumors of the middle ear. Ann Otol Rhinol Laryngol 99: 547-552, 1990.

7. Inoue S, Tanaka K and Kannae S: Primary carcinoid tumor of the ear. Virchows Arch A Pathol Anat Histol 396: 357-363, 1982.

8. Ohta Y, Morihana T, Hanada Y, Imai T, Iwamoto Y, Uno A, Kawashima T, Hasegawa T, Kitahara T, Morii E and Inohara H: A clinical study of 61 cases of tumorous disease in external ear canal: Diagnosis of tumor. Otol Jpn 25: 771-776, 2015

9. Mohan H, Handa U, Amanjit, Kotwal SA and Dass A: Adenoid cystic carcinoma of the external auditory canal. A case report with diagnosis by fine needle aspiration. Acta Cytol 47: 792-794, 2003.

10. Murphy GF, Pilch BZ, Dickersin GR, Goodman ML and Nadol JB Jr: Carcinoid tumor of the middle ear. Am J Clin Pathol 73: 816-823, 1980 
11. Torske KR and Thompson LD: Adenoma versus carcinoid tumor of the middle ear: A Study of 48 cases and review of the literature. Mod Pathol 15: 543-555, 2002.

12. Renshaw AA, Haja J, Lozano RL and Wilbur DC; Cytology Committee, College of American Pathologists: Distinguishing carcinoid tumor from small cell carcinoma of the lung: Correlating cytologic features and performance in the College of American Pathologists Non-Gynecologic Cytology Program. Arch Pathol Lab Med 129: 614-618, 2005.

13. Burke AP, Thomas RM, Elsayed AM and Sobin LH: Carcinoids of the jejunum and ileum: An immnohistochemical and climicopathologic study of 167 cases. Cancer 79: 1086-1093, 1997.

14. Sato K, Higaki Y, Sakaguchi S, Hirano M, Tanimura A and Sasaguri Y: Carcinoid tumor of the larynx. Auris Nasus Larynx 18: 39-53, 1991.

15. Manni JJ, Faverly DR and Van Haelst UJ: Primary carcinoid tumors of the middle ear. Report on four cases and review of the literature. Arch Otolaryngol Head Neck Surg 118: 1341-1347, 1992.
16. Knerer B, Matula C, Youssefzadeh S, Ulrich W and Swoboda H: Treatment of a local recurrence of a carcinoid tumor of the middle ear by extended subtotal petrosectomy. Eur Arch Otorhinolaryngol 255: 57-61, 1998

17. Inzani F, Petrone G, Fadda S and Rindi G: Cyto-histology in NET: What is necessary today and what is the future. Rev Endocr Metab Disore, 2017. Doi: 10.1007/s11154-017-9428-x.

18. Biancosino C, Krüger M, Vollmer E and Welker L: Intraoperative fine needle apirations-dignosis and typing of lung cancer in small biopsies: Challenges and limitations. Diagn Pathol 11: 59, 2016. 